\title{
The use of extracorporeal membrane oxygenation in the anticipated difficult airway: a case report and systematic review
}

\section{Recours à l'oxygénation extracorporelle en prévision de la gestion de voies respiratoires difficiles : rapport de cas et étude systématique}

\author{
Gemma Malpas, MBChB, FANZCA · Orlando Hung, MD, FRCPC · Ainslie Gilchrist, MD • \\ Chrison Wong, MD • Blaine Kent, MD, FRCPC • Gregory M. Hirsch, MD, FRSCS • \\ Robert D. Hart, MD FRSCS
}

Received: 29 October 2017/Revised: 18 December 2017/Accepted: 20 December 2017/Published online: 1 March 2018 (C) Canadian Anesthesiologists' Society 2018

\begin{abstract}
While extracorporeal membrane oxygenation $(E C M O)$ is an effective method of oxygenation for patients with respiratory failure, further refinement of its incorporation into airway guidelines is needed. We present a case of severe glottic stenosis from advanced thyroid carcinoma in which gas exchange was facilitated by venoarterial ECMO prior to achieving a definitive airway. We also conducted a systematic review of the MEDLINE, EMBASE, CINAHL, and Web of Science databases, using the keywords "airway/ tracheal obstruction", "anesthesia", "extracorporeal", and "cardiopulmonary bypass" to identify reports where ECMO was initiated as the a priori method of oxygenation during difficult airway management.Thirty-six papers were retrieved discussing
\end{abstract}

Prior poster presentations at the Society for Airway Management conference, Newport Beach, California, 16 September 2017, and ePoster presentation at the American Society of Anesthesiologists' annual meeting, Boston, Massachusetts, 22 October 2017.

G. Malpas, MBChB, FANZCA ( $\square)$ - O. Hung, MD, FRCPC . A. Gilchrist, MD - C. Wong, MD - B. Kent, MD, FRCPC Department of Anesthesia, Pain Management and Perioperative Medicine, Queen Elizabeth II Health Sciences, Dalhousie University, 1278 South Park St, Halifax, NS B3H 2Y9, Canada e-mail: gmalpas@icloud.com

G. M. Hirsch, MD, FRSCS

Division of Cardiac surgery, Department of Surgery, Dalhousie University, Halifax, NS, Canada

R. D. Hart, MD FRSCS

Division of Otolaryngology-Head and Neck Surgery, Department of Surgery, Dalhousie University, Halifax, NS, Canada the use of ECMO or cardiopulmonary bypass $(C P B)$ for the management of critical airway obstruction. Forty-five patients underwent pre-induction of anesthesia institution of CPB or ECMO for airway obstruction. The patients presenting with critical airway obstruction had a range of airway pathologies with tracheal tumours (31\%), tracheal stenosis (20\%), and head and neck cancers (20\%) being the most common. All cases reported a favourable patient outcome with all patients surviving to hospital discharge without significant complications. While most practitioners are familiar with the fundamental airway techniques of bag-mask ventilation, supraglottic airway use, tracheal intubation, and front-of-neck airway access for oxygenation, these techniques have limitations in managing patients with pre-existing severe airway obstruction. The use of ECMO should be considered in patients with severe (or near-complete) airway obstruction secondary to anterior neck or tracheal disease. This approach can provide essential tissue oxygenation while attempts to secure a definitive airway are carried out in a controlled environment.

Résumé L'oxygénation par membrane extracorporelle (ECMO) est une méthode d'oxygénation efficace chez les patients présentant une insuffisance respiratoire, mais il est nécessaire de mieux préciser son inclusion dans les lignes directrices concernant la gestion des voies respiratoires. Nous présentons un cas de sténose sévère de la glotte due à un carcinome de la thyrö̈de dans lequel l'échange gazeux était facilité par une ECMO veino-artérielle avant la réalisation d'un accès des voies respiratoires définitif. Nous avons aussi effectué une revue systématique des bases 
de données MEDLINE, EMBASE, CINAHL et Web of Science en utilisant les mots clés "obstruction des voies respiratoires/de la trachée ", "anesthésie », " extracorporelle " et "circulation extracorporelle " pour identifier des cas dans lesquels une ECMO a été mise en place comme méthode d'oxygénation a priori au cours de la gestion de voies respiratoires difficiles. Trentesix articles discutant de l'utilisation de l'oxygénation ou de la circulation extracorporelle dans la gestion des obstructions majeures des voies respiratoires ont été retenus. Quarante-cinq patients ont subi une préinduction d'anesthésie par oxygénation ou circulation extracorporelle en raison d'une obstruction des voies respiratoires. Les patients présentant une obstruction majeure des voies respiratoires étaient principalement des patients atteints de tumeurs de la trachée (31\%), sténose de la trachée $(20 \%)$ et de cancers de la tête et du cou (20\%). Dans tous les cas, l'évolution des patients a été favorable: ils ont pu quitter l'hôpital en vie et sans complications significatives. Bien que la majorité des praticiens connaissent les techniques de base de ventilation au masque et au ballon, l'utilisation des voies respiratoires supraglottiques et l'accès cervical antérieur des voies respiratoires pour assurer une oxygénation correcte, ces techniques ont des limites dans la gestion de patients ayant une obstruction préexistante sévère des voies respiratoires. L'utilisation de l'oxygénation extracorporelle devrait être envisagée chez les patients ayant une obstruction sévère (ou quasi complète) des voies respiratoires à la suite d'une maladie de la trachée ou du segment antérieur du cou. Cette approche peut procurer une oxygénation tissulaire essentielle pendant que des tentatives visant à assurer un accès définitif des voies respiratoires sont menées dans un environnement contrôlé.

Guidelines by the American Society of Anesthesiologists, ${ }^{1-3}$ The Difficult Airway Society of Great Britain and Ireland, ${ }^{4,5}$ and the Canadian Airway Focus Group (CAFG) $)^{6,7}$ emphasize the use of four fundamental techniques of sustaining adequate tissue oxygenation: bag-mask ventilation (BMV), supraglottic airway (SGAs), tracheal intubation, ${ }^{8}$ and the front-of-neck airway. ${ }^{9}$ Airway obstructions, particularly at or below the level of the glottis, can present significant challenges beyond the above guidelines. While extracorporeal membrane oxygenation (ECMO) is an effective means of providing adequate oxygenation for patients with respiratory failure, ${ }^{10-12}$ further development involving possible incorporation into airway guidelines is required.
Extracorporeal membrane oxygenation is a life-support technique using mechanical devices to support both cardiac and respiratory function when the native systems fail. ${ }^{13}$ Two forms of ECMO exist: veno-arterial (VA) ECMO, which has the potential to provide complete respiratory and hemodynamic support, and veno-venous (VV) ECMO, which supports the respiratory system alone, allowing gas exchange outside the body. ${ }^{14}$ Dorson $e t$ al. first reported the use of a membrane oxygenator for cardiopulmonary bypass in infants in $1969 ;{ }^{15}$ following that, ECMO was successfully used as support in infants with congenital heart defects undergoing cardiac surgery. ${ }^{16}$ The use of both forms of ECMO in adult patients only began to flourish after the publication of the randomized-controlled trial comparing conventional ventilatory support $v s$ ECMO for severe adult respiratory failure in $2009 .{ }^{17}$ This trial reported a significant improvement in the mortality rate without severe disability at six months in patients with severe respiratory disease who were transferred to a specialist centre for consideration for ECMO treatment compared with continued conventional ventilation. Since then, the use of either form of ECMO has been used for a wide range of conditions that require cardiac and/ or respiratory support, ${ }^{16,18,19}$ as well as for support during a range of cardiac and thoracic ${ }^{20}$ interventions.

The objective of this report is to present a case of advanced thyroid carcinoma causing severe glottic and proximal tracheal obstruction in which adequate gas exchange was facilitated by ECMO prior to achieving a definitive airway under total intravenous anesthesia. This case serves to illustrate the use of ECMO in the obstructed airway, when the previously mentioned four fundamental techniques of oxygenation are likely to fail. This less understood technique for management of gas exchange in the difficult airway setting was then systematically reviewed.

\section{Case presentation}

A 77-yr-old male (who consented to this report) presented to the preoperative anesthesia clinic as an urgent outpatient consult from the ear, nose, and throat (ENT) service.

On presentation, the patient was stridorous at rest and only able to ambulate less than a few steps. He stated that the stridor had been increasing over the past few weeks and that he was no longer able to sleep flat in bed. He had been seen in the emergency department earlier in the week and given a short course of oral prednisone $30 \mathrm{mg}$ daily for airway edema, but he did not feel it had made a significant improvement.

The patient had a complex cardiac history consisting of coronary artery bypass graft surgery in 2003, with pre- 
existing pacemaker-dependent complete heart block, with subsequent infection at his pacemaker site, requiring removal and replacement with a right infraclavicular pacemaker. Echocardiography in 2015 reported normal biventricular function with no suggestion of elevated pulmonary artery pressures. He denied any recent chest pain, but, because of his increasing difficulty with breathing, had not been able to undertake any significant activity for the past four months.

Physical examination revealed an elderly obese male with a body mass index of $35 \mathrm{~kg} \cdot \mathrm{m}^{-2}$, severe stridor, sitting upright, using his accessory muscles of respiration. His oxygen saturation on room air was $95 \%$.

A computed tomographic (CT) view of the head and neck was performed, which revealed a 1-mm opening at the level of the glottis (Fig. 1). Previous anesthetic records showed a Cormack-Lehane grade 1 view using a Macintosh laryngoscope. The remainder of his history and investigations was unremarkable.

Consultation among the anesthesia and ENT specialists followed. Due to the anticipated difficulties with BMV, SGA, and tracheal intubation due to both the distorted anatomy and very small tracheal lumen, these were all dismissed as viable options. A front-of-neck airway access under local anesthesia was also excluded as an option because of the extensive nature of the thyroid carcinoma distorting the anatomy and the ability of the patient to tolerate the supine position. A cardiac surgeon was then consulted about the possibility of instituting ECMO in the awake state to allow for oxygenation before securing a definitive airway under total intravenous anesthesia. He was admitted to the ENT ward for intravenous steroids.

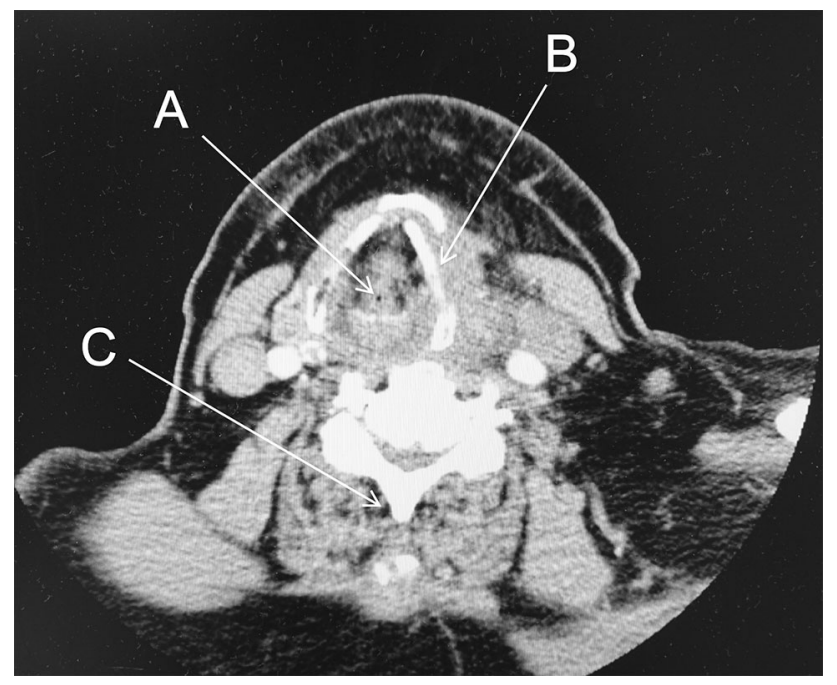

Fig. 1 Coronal view showing 1-mm internal airway diameter. (A: 1$\mathrm{mm}$ Subglottic airway; B: left thyroid ala; C: C5 spinous process)
Heliox (a mixture of helium and oxygen) was administered through a non-rebreathing face mask prior to surgery.

Preoperative CT angiography was performed to assess the arterial and venous access sites for ECMO and showed significant calcific atherosclerotic disease of both femoral arteries.

With the heliox continuing, the patient was taken to the operating room (OR) and transferred to an OR table in a semi-recumbent position where routine monitors were placed in addition to a radial arterial cannula, processed EEG, and non-invasive near-infrared spectroscopy (NIRS) tissue oximetry (INVOS ${ }^{\mathrm{TM}}$, Medtronic, Minneapolis, MN, USA).

Following positioning and placement of monitoring, an airway strategy was communicated to the OR personnel. The strategy consisted of insertion of the ECMO cannulae under local anesthesia and establishing ECMO flow. Once established, induction of anesthesia would occur. Following induction of anesthesia, the anesthesiologist would undergo one attempt at tracheal intubation with a video-laryngoscope using a styletted micro-laryngoscopy tube (MLT). If this was unsuccessful, the surgeon would consider an attempt at rigid bronchoscopy prior to proceeding to tracheotomy through the thyroid carcinoma if that were to fail. Following endotracheal intubation, ECMO would then be weaned preventing the need for continued anticoagulation.

The patient's right-sided pacemaker precluded the use of the axillary vessels, and the planned thyroidectomy and laryngectomy precluded access to the internal jugular vessels. Due to the anticipated time required to achieve exposure of the femoral vessels resulting from the patient's body habitus and semi-recumbent position, the decision was made to establish ECMO via unilateral femoral vessels. Given the clinical indication, VV-ECMO would have been the preferred ECMO mode because of the sole need for oxygenation and the reduced requirement for anticoagulation. Nevertheless, the anatomical and premorbid conditions, as well as the availability of equipment, led to VA-ECMO being selected by the cardiac surgeon as the most suitable ECMO mode.

After sedation with midazolam $0.5 \mathrm{mg} i v$, the right groin was prepped, draped, and infiltrated with lidocaine. After the administration of heparin 5,000 units $i v$ and the postheparin activated clotting time confirmed adequate anticoagulation, a femoral arterial perfusion cannula was placed and secured into position. The femoral vein was then cannulated and attached to the ECMO circuit with its position in the right atrium confirmed by transthoracic echocardiography following which full ECMO flow was incrementally instituted.

Anesthesia was then induced and maintained with propofol/remifentanil and rocuronium for neuromuscular 
blockade. Using video Macintosh laryngoscopy (C-MAC \#4 blade; Karl Storz Endoscopy, Culver City, CA, USA), the glottis was easily visualized but appeared edematous and stenotic. One attempt at intubation with a styletted 5.0$\mathrm{mm}$ internal diameter (ID) MLT was made but was unsuccessful because of an inability to advance beyond the solid tumour at the glottic opening. In discussion with the ENT surgeon, it was elected to proceed to tracheotomy and forgo any attempt at rigid bronchoscopy.

Saturations of $72-80 \%$ were recorded by the pulse oximeter (right index finger) likely related to the fact that ECMO was providing highly oxygenated blood to the lower extremity while poorly oxygenated blood was being ejected into the ascending aorta. Despite this, cerebral oximetry as measured by NIRS was acceptable at $72 \%$, perhaps owing to sufficient super-oxygenated blood supply flowing up the descending aorta and mixing sufficiently at the level of the distal aortic arch.

The tracheotomy, though technically challenging because of the presence of the large anterior obstructive mass, allowed placement of a reinforced 7.0-mm ID endotracheal tube (ETT) (Mallinckrodt ${ }^{\mathrm{TM}}$ Lo-Contour Reinforced Tracheal Tube, Covidien, Minneapolis, MN, USA). Following tracheal intubation, anesthesia was transitioned to an inhalational anesthetic using sevoflurane. The ECMO was then weaned and the femoral vessels were decannulated. Heparin was reversed using protamine prior to proceeding with the thyroidectomy, laryngectomy, and central lymph node dissection.

At the conclusion of the surgery, the 7.0-mm ID reinforced ETT was replaced by an 8.0-mm ID Shiley tracheostomy tube (Covidien, Minneapolis, MN, USA) and the patient was taken to the intensive care unit for overnight monitoring followed by transfer to the ENT ward, where he remained until discharge home the following week.

The tissue pathology confirmed an aggressive multifocal papillary thyroid carcinoma with high-grade transformation, predominantly to squamous cell cancer, and focal insular carcinoma. Central neck nodes were negative for malignancy.

Systematic review methods

We conducted electronic literature searches for all published articles up until September 2017 from the MEDLINE, EMBASE, CINAHL, and Web of Science databases using the key words "airway obstruction", "airway management", "tracheal obstruction", "CPB/heart lung bypass/cardiopulmonary bypass", "anesthesia/anaesthesia", and "ECMO/extracorporeal membrane oxygenation" (see Appendix for details).
Reference lists of the selected articles were also searched for additional papers. Published meeting abstracts were included (if all inclusion criteria were addressed and met) and publications in languages other than English were only included if all inclusion criteria were fulfilled by the English-language abstract.

All case reports and observational studies reporting the use of ECMO in adults ( $>18 \mathrm{yr}$ ) for airway management were considered and individually evaluated. For the purpose of this study, we only selected cases in which extracorporeal life support (ECMO or CPB) was initiated as the a priori method of oxygenation and not as a rescue technique following a failed intubation, failed ventilation, or cardiorespiratory arrest. This was determined following review of titles meeting the initial search criteria. All patients included in this review had ECMO or CPB cannulae inserted and flows established prior to airway intervention. Patients were included if ECMO or CPB was used as a substitute to definitive airway management.

Articles describing the use of ECMO for patients with deteriorating circulation or primarily cardiac support were excluded. Information on the authors, institution, population, and dates was checked to identify duplicate publications. Duplicated patients in consecutive reports from the same institution or author were excluded.

All cases were then analyzed and tabulated, displaying the indication for ECMO, the condition of the patient prior to ECMO initiation, the mode and duration of ECMO, the pathology leading to airway obstruction, the definitive management of the patient, and the clinical outcome.

\section{Results}

Literature search

Our search yielded 784 titles with a further five additional records identified through the reference lists. Following the removal of duplicates, 621 records were screened for inclusion criteria (Fig. 2). Five hundred and thirty-five records were removed, leading to 86 abstracts considered appropriate for full-text evaluation. There were no randomized-controlled trials on the use of ECMO for severe airway obstruction.

\section{Study characteristics}

Thirty-six papers published between 1976 and 2017 discussing cases utilizing ECMO or CPB for the management of critical airway obstruction were included in the review, including 28 case reports and eight case series. Within these case series, only select cases met 


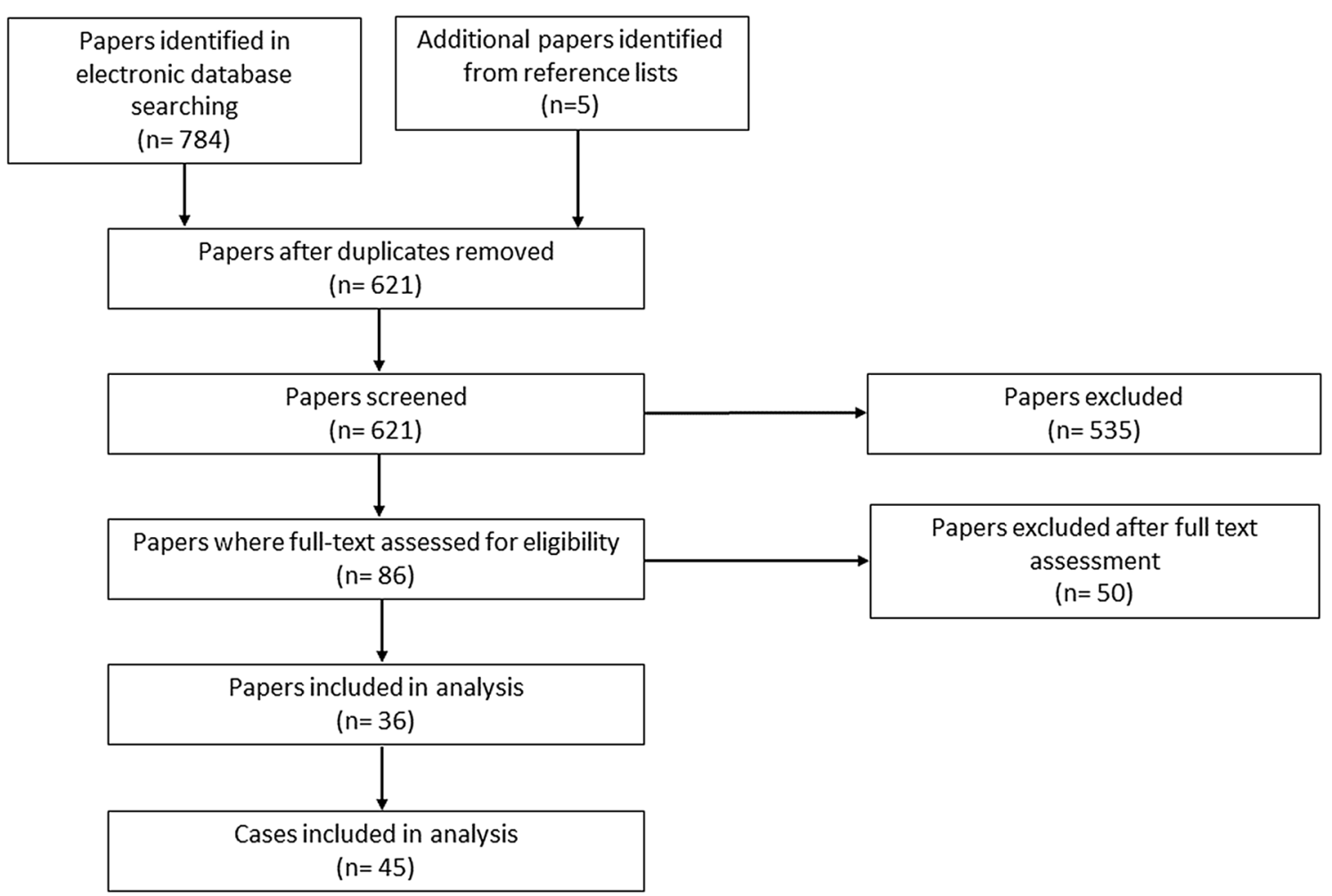

Fig. 2 Bibliographic search of the literature

criteria for inclusion in the review. ${ }^{21}$ One included paper was published in Japanese, ${ }^{29}$ and one published only as an abstract, ${ }^{30}$ but these abstracts revealed sufficient clinical information to be included in the review. The most frequent reasons for exclusion were failing the predetermined inclusion criteria, patients $<18 \mathrm{yr}$, and inability to obtain detailed patient information. Eight papers reported a case series in which ECMO was used in airway management. A total of 45 patients are included in this review. Results are summarized in the Table.

The patients presenting with critical airway obstruction had a range of airway pathologies. These included: tracheal tumour (14 patients, 31\%), 22,24,27,28,31 tracheal stenosis (nine patients, 20\%), ${ }^{21,22,38,39}$ head and neck cancer (nine patients, 20\%), ${ }^{23,29,40}$ large mediastinal mass (six patients, $13 \%),{ }^{47}$ primary lung cancer (two patients, 5\%), ${ }^{25,30}$ benign thyroid goiter (two patients, 5\%), ${ }^{53,54}$ esophageal cancer (one patient, 2\%), ${ }^{25}$ malignant melanoma (one patient, 2\%), ${ }^{55}$ and tracheal granulomas (one patient, $2 \%){ }^{56}$ All surgeries included in this review were performed in tertiary institutions with ready access to CPB or ECMO. Sixteen patients underwent full CPB (as opposed to only ECMO) in reports up until 2014.

All published cases reported a favourable patient outcome with all patients surviving to hospital discharge without significant complications. In 2015, Kim et al. ${ }^{21}$ published a case series of 15 patients who underwent pre- induction ECMO for airway obstruction. They reported no mortality associated with the elective use of ECMO during the management of severe airway disease; however, deaths due to hypoxic brain damage were reported when non $a$ priori ECMO was only considered following failed airway management. ${ }^{21}$ Many case series reported their complications as a cumulative composite semiquantitative statement (e.g., low) and without detailed information about the types and severity of complications.

\section{Discussion}

The incidence of upper airway tumours in Canada has increased over the past 30 years, with an estimated 18.5 people per 100,000 diagnosed with thyroid cancer in $2016 .{ }^{57}$ Early detection and intervention for these tumours has the potential to reduce the incidence of severe airway obstruction. On the other hand, patients who present late with these tumours may show signs of airway obstruction.

From our search, 36 articles were retrieved that discussed the use of ECMO/CPB for the urgent management of critical airway obstruction. Eighteen patients underwent pre-induction VV- ECMO and two patients VA-ECMO, 24 patients had CPB, and in one patient the support mode was unspecified. Patients with critical airway disease presented with a range of airway 
Table Case reports of elective ECMO utilization in airway management

\begin{tabular}{|c|c|c|c|c|c|c|c|c|c|}
\hline First author & Year & Age & $\begin{array}{l}\text { Condition } \\
\text { prior to } \\
\text { ECMO }\end{array}$ & $\begin{array}{l}\text { ECMO } \\
\text { mode }\end{array}$ & ECMO duration & Obstruction type & Comments & $\begin{array}{l}\text { Definitive } \\
\text { management }\end{array}$ & Final result \\
\hline Coles $^{46}$ & 1976 & $\mathrm{~F} / 25$ & $\begin{array}{l}\text { Acute } \\
\text { respiratory } \\
\text { distress }\end{array}$ & $\mathrm{CPB}$ & $\begin{array}{l}\text { Until lower } \\
\text { tracheal } \\
\text { exposure }\end{array}$ & $\begin{array}{l}\text { Tracheal } \\
\quad \text { carcinoma }\end{array}$ & $\begin{array}{l}\text { Near complete } \\
\text { obstruction }\end{array}$ & Resection & Discharged \\
\hline Bricker $^{22}$ & 1979 & $\mathrm{~F} / 68$ & $\begin{array}{l}\text { Stridor and } \\
\text { choking } \\
\text { spells }\end{array}$ & CPB & Operative case & Tracheal tumour & $\begin{array}{l}\text { Near complete } \\
\text { occlusion }\end{array}$ & $\begin{array}{l}\text { Tracheal } \\
\text { resection } \\
\text { and } \\
\text { anastomosis }\end{array}$ & Discharged \\
\hline Bricker $^{22}$ & 1979 & $\mathrm{M} / 54$ & $\begin{array}{l}\text { Dyspnea, } \\
\text { stridor, and } \\
\text { orthopnea }\end{array}$ & $\mathrm{CPB}$ & Operative case & $\begin{array}{r}\text { Traumatic } \\
\text { tracheal } \\
\text { stenosis }\end{array}$ & $\begin{array}{l}2-3 \mathrm{~mm} \\
\text { diameter }\end{array}$ & $\begin{array}{l}\text { Tracheal } \\
\text { resection } \\
\text { and } \\
\text { anastomosis }\end{array}$ & Discharged \\
\hline Jensen $^{32}$ & 1983 & $\mathrm{~F} / 65$ & $\begin{array}{c}\text { Dyspnea and } \\
\text { orthopnea }\end{array}$ & CPB & Operative case & Tracheal tumour & $\begin{array}{l}\text { Near complete } \\
\text { occlusion }\end{array}$ & Tracheotomy & Discharged \\
\hline Wilson $^{31}$ & 1984 & $\mathrm{M} / 47$ & $\begin{array}{l}\text { Severe } \\
\quad \text { dyspnea }\end{array}$ & $\mathrm{CPB}$ & Operative case & $\begin{array}{l}\text { Squamous cell } \\
\text { carcinoma of } \\
\text { the trachea }\end{array}$ & $\begin{array}{l}\text { Near complete } \\
\text { occlusion }\end{array}$ & $\begin{array}{l}\text { Surgical } \\
\quad \text { resection }\end{array}$ & Discharged \\
\hline Hicks $^{54}$ & 1986 & $\mathrm{~F} / 70$ & $\begin{array}{l}\text { Dyspnea and } \\
\text { stridor }\end{array}$ & CPB & $\begin{array}{l}\text { Until definitive } \\
\text { airway }\end{array}$ & Substernal goitre & 4-mm airway & $\begin{array}{l}\text { Goitre } \\
\quad \text { resection }\end{array}$ & Discharged \\
\hline $\operatorname{Rosa}^{44}$ & 1996 & $\mathrm{~F} / 51$ & $\begin{array}{l}\text { Respiratory } \\
\text { distress and } \\
\text { stridor }\end{array}$ & СРB & Operative case & $\begin{array}{l}\text { Thyroid } \\
\text { lymphoma }\end{array}$ & & $\begin{array}{l}\text { Tracheotomy, } \\
\text { resection }\end{array}$ & Discharged \\
\hline Belmont $^{43}$ & 1998 & $\mathrm{~F} / 73$ & $\begin{array}{l}\text { Dyspnea, } \\
\text { hypoxia, } \\
\text { stridor, and } \\
\text { orthopnea }\end{array}$ & $\mathrm{CPB}$ & Operative case & $\begin{array}{l}\text { Thyroid } \\
\text { lymphoma }\end{array}$ & $\begin{array}{l}\text { Near complete } \\
\text { obstruction }\end{array}$ & Tracheotomy & Discharged \\
\hline Onozawa $^{29}$ & 1999 & M/64 & $\begin{array}{c}\text { Dyspnea and } \\
\text { orthopnea }\end{array}$ & VA & Operative case & $\begin{array}{l}\text { Thyroid } \\
\text { carcinoma }\end{array}$ & $\begin{array}{l}\text { 5-mm tracheal } \\
\text { diameter }\end{array}$ & $\begin{array}{l}\text { Tracheotomy } \\
\text { and } \\
\text { resection }\end{array}$ & Discharged \\
\hline Tempe $^{52}$ & 2001 & $\mathrm{M} / 22$ & $\begin{array}{l}\text { Severe } \\
\text { dyspnea, } \\
\text { respiratory } \\
\text { distress }\end{array}$ & $\mathrm{CPB}$ & $6 \min$ & Liposarcoma & $\begin{array}{l}\text { Airway } \\
\text { compression }\end{array}$ & $\begin{array}{l}\text { Surgical } \\
\quad \text { resection }\end{array}$ & Discharged \\
\hline $\mathrm{Chiu}^{38}$ & 2003 & $F / 27$ & $\begin{array}{l}\text { Tracheotomy, } \\
\text { severe } \\
\text { stridor, and } \\
\text { respiratory } \\
\text { distress }\end{array}$ & CPB & $\begin{array}{l}\text { Until } \\
\text { endobronchial } \\
\text { intubation }\end{array}$ & $\begin{array}{l}\text { Post-intubation } \\
\text { tracheal } \\
\text { stenosis }\end{array}$ & $\begin{array}{l}\text { 3-cm-long } \\
\text { stenotic } \\
\text { region }\end{array}$ & $\begin{array}{r}\text { Resection and } \\
\text { anastomosis }\end{array}$ & Discharged \\
\hline Shiraishi $^{25}$ & 2004 & $\mathrm{M} / 57$ & $\begin{array}{l}\text { Severe } \\
\text { dyspnea }\end{array}$ & VV & Operative case & $\begin{array}{l}\text { Non-small-cell } \\
\text { lung carcinoma }\end{array}$ & $\begin{array}{l}\text { Completely } \\
\text { obstructed } \\
\text { RMB with } \\
\text { severe } \\
\text { obstruction } \\
\text { at the carina } \\
\text { and the } \\
\text { LMB }\end{array}$ & $\begin{array}{l}\text { Dynamic stent } \\
\text { placement }\end{array}$ & Discharged \\
\hline Shiraishi $^{25}$ & 2004 & $\mathrm{~F} / 59$ & $\begin{array}{l}\text { Severe } \\
\text { dyspnea }\end{array}$ & VV & Operative case & $\begin{array}{l}\text { Advanced } \\
\text { esophageal } \\
\text { carcinoma }\end{array}$ & $\begin{array}{l}\text { Severe lower } \\
\text { tracheal and } \\
\text { carinal } \\
\text { obstruction }\end{array}$ & $\begin{array}{l}\text { Stent } \\
\text { placement }\end{array}$ & Discharged \\
\hline Goyal $^{35}$ & 2005 & $\mathrm{~F} / 31$ & $\begin{array}{l}\text { Weak voice } \\
\text { and stridor }\end{array}$ & $\mathrm{CPB}$ & Operative case & $\begin{array}{l}\text { Adenoid cystic } \\
\text { carcinoma }\end{array}$ & $\begin{array}{l}\text { Near complete } \\
\text { obstruction }\end{array}$ & $\begin{array}{l}\text { Tumour } \\
\text { resection }\end{array}$ & Discharged \\
\hline Weinbroum $^{45}$ & 2005 & $\mathrm{~F} / 70$ & $\begin{array}{c}\text { Stridor and } \\
\text { dyspnea }\end{array}$ & $\mathrm{CPB}$ & Until intubation & $\begin{array}{l}\text { Thyroid } \\
\text { lymphoma }\end{array}$ & $\begin{array}{l}\text { 1-mm patent } \\
\text { airway }\end{array}$ & Tracheotomy & Not stated \\
\hline Tyagi $^{26}$ & 2006 & $\mathrm{M} / 27$ & $\begin{array}{l}\text { Cyanosis, } \\
\text { respiratory } \\
\text { distress }\end{array}$ & $\mathrm{CPB}$ & $25 \mathrm{~min}$ & SCC trachea & $\begin{array}{l}>90 \% \\
\quad \text { occlusion }\end{array}$ & $\begin{array}{l}\text { Surgical } \\
\quad \text { resection }\end{array}$ & Not stated \\
\hline
\end{tabular}


Table continued

\begin{tabular}{|c|c|c|c|c|c|c|c|c|c|}
\hline First author & Year & Age & $\begin{array}{l}\text { Condition } \\
\text { prior to } \\
\text { ECMO }\end{array}$ & $\begin{array}{l}\text { ECMO } \\
\text { mode }\end{array}$ & ECMO duration & Obstruction type & Comments & $\begin{array}{l}\text { Definitive } \\
\text { management }\end{array}$ & Final result \\
\hline Tyagi $^{26}$ & 2006 & $\mathrm{~F} / 31$ & $\begin{array}{l}\text { Cyanosis, } \\
\text { respiratory } \\
\text { distress }\end{array}$ & CPB & $25 \mathrm{~min}$ & $\begin{array}{l}\text { Adenoid cystic } \\
\text { carcinoma }\end{array}$ & $\begin{array}{l}\text { Approx. } 90 \% \\
\text { occlusion }\end{array}$ & $\begin{array}{l}\text { Surgical } \\
\quad \text { resection }\end{array}$ & Not stated \\
\hline Soon $^{51}$ & 2007 & $\mathrm{~F} / 52$ & $\begin{array}{l}\text { Dyspnea on } \\
\text { exertion }\end{array}$ & CPB & $\begin{array}{l}48 \text { min } \\
\text { (induction and } \\
\text { establishment } \\
\text { of ventilation) }\end{array}$ & Thymoma & $\begin{array}{l}\text { Severe airway } \\
\text { compression }\end{array}$ & $\begin{array}{l}\text { Surgical } \\
\text { debulking }\end{array}$ & Discharged \\
\hline Zhou $^{27}$ & 2007 & $\mathrm{M} / 25$ & $\begin{array}{l}\text { Respiratory } \\
\text { distress }\end{array}$ & CPB & $\begin{array}{l}64 \text { min } \\
\text { (operative } \\
\text { case) }\end{array}$ & $\begin{array}{l}\text { Benign } \\
\text { hypervascular } \\
\text { leiomyoma }\end{array}$ & $\begin{array}{l}\text { 1-mm tracheal } \\
\text { lumen }\end{array}$ & $\begin{array}{l}\text { Surgical } \\
\quad \text { resection }\end{array}$ & Discharged \\
\hline $\mathrm{Liu}^{24}$ & 2009 & Adult & $\begin{array}{l}\text { Severe } \\
\text { dyspnea }\end{array}$ & CPB & $25 \min$ & Tracheal tumour & $\begin{array}{l}\text { Near complete } \\
\text { obstruction }\end{array}$ & Resection & Unknown \\
\hline $\mathrm{Liu}^{24}$ & 2009 & Adult & $\begin{array}{l}\text { Severe } \\
\text { dyspnea }\end{array}$ & CPB & $30 \mathrm{~min}$ & Tracheal tumour & $\begin{array}{l}\text { Near complete } \\
\text { obstruction }\end{array}$ & Resection & Unknown \\
\hline $\mathrm{Shao}^{53}$ & 2009 & $\mathrm{~F} / 51$ & Dyspnea & VA & $\begin{array}{l}7 \mathrm{hr} 35 \mathrm{~min} \\
\text { (operative } \\
\text { case) }\end{array}$ & $\begin{array}{l}\text { Multi-nodular } \\
\text { thyroid goitre } \\
\text { and rheumatoid } \\
\text { arthritis }\end{array}$ & 5-mm diameter & & Discharged \\
\hline Jeon $^{40}$ & 2009 & $\mathrm{~F} / 68$ & $\begin{array}{l}\text { Dyspnea, } \\
\text { voice } \\
\text { change, } \\
\text { intermittent } \\
\text { hemoptysis }\end{array}$ & VV & Operative case & $\begin{array}{l}\text { Thyroid } \\
\text { carcinoma }\end{array}$ & $\begin{array}{l}\text { Near complete } \\
\text { occlusion }\end{array}$ & & Discharged \\
\hline Sendasgupta ${ }^{47}$ & 2010 & $\mathrm{M} / 65$ & $\begin{array}{l}\text { Severe } \\
\text { dyspnea, } \\
\text { stridor, } \\
\text { cyanosis }\end{array}$ & CPB & Operative case & $\begin{array}{l}\text { Anterior } \\
\text { mediastinal } \\
\text { tumour }\end{array}$ & $\begin{array}{l}\text { 7-mm tracheal } \\
\text { diameter } \\
\text { with } \\
\text { distortion }\end{array}$ & $\begin{array}{l}\text { Surgical } \\
\quad \text { resection }\end{array}$ & Discharged \\
\hline Mehta $^{30}$ & 2011 & $\mathrm{~F} / 46$ & $\begin{array}{l}\text { Respiratory } \\
\text { distress }\end{array}$ & Unspecified & Operative case & $\begin{array}{l}\text { Non-small-cell } \\
\text { lung carcinoma }\end{array}$ & 2-mm diameter & Stent insertion & Discharged \\
\hline Yang $^{42}$ & 2012 & $\mathrm{M} / 48$ & $\begin{array}{l}\text { Orthopnea and } \\
\text { hemoptysis }\end{array}$ & $\mathrm{CPB}$ & Operative case & $\begin{array}{l}\text { Thyroid } \\
\text { carcinoma }\end{array}$ & $\begin{array}{c}\text { Near complete } \\
\text { obstruction }\end{array}$ & Tracheal stent & Discharged \\
\hline Gourdin $^{56}$ & 2012 & $\mathrm{M} / 27$ & $\begin{array}{l}\text { Stridor and } \\
\text { severe } \\
\text { halitosis }\end{array}$ & VV & $\begin{array}{l}120 \text { min } \\
\text { (operative } \\
\text { case) }\end{array}$ & $\begin{array}{l}\text { Tracheal stent } \\
\text { occlusions and } \\
\text { granulomas }\end{array}$ & $\begin{array}{l}\text { 4.9-mm } \\
\text { diameter }\end{array}$ & $\begin{array}{l}\text { Stent removal } \\
\text { and } \\
\text { replacement }\end{array}$ & Discharged \\
\hline $\mathrm{Gao}^{37}$ & 2013 & $\mathrm{~F} / 51$ & $\begin{array}{l}\text { Severe } \\
\text { dyspnea }\end{array}$ & CPB & $\begin{array}{l}15 \min \\
\quad \text { (intubation) }\end{array}$ & Tracheal tumour & $80 \%$ occlusion & $\begin{array}{l}\text { Tracheal } \\
\text { resection } \\
\text { and } \\
\text { anastomosis }\end{array}$ & Discharged \\
\hline Hong $^{23}$ & 2013 & M78 & Unspecified & VV & $\begin{array}{l}2.2 \mathrm{hr} \text { (Operative } \\
\text { case) }\end{array}$ & $\begin{array}{l}\text { Head and neck } \\
\text { cancer }\end{array}$ & $\begin{array}{l}\text { Case series, } \\
\text { however } \\
\text { only } 1 \\
\text { patient met } \\
\text { criteria }\end{array}$ & & Discharged \\
\hline $\operatorname{Erden}^{48}$ & 2014 & $\mathrm{M} / 73$ & $\begin{array}{l}\text { Respiratory } \\
\text { distress }\end{array}$ & CPB & Operative case & $\begin{array}{l}\text { External } \\
\text { mediastinal } \\
\text { mass }\end{array}$ & $\begin{array}{l}\text { 1-mm tracheal } \\
\text { stenosis }\end{array}$ & $\begin{array}{l}\text { Stent } \\
\text { placement }\end{array}$ & Not stated \\
\hline Said $^{49}$ & 2014 & $\mathrm{~F} / 37$ & $\begin{array}{l}\text { Dyspnea, } \\
\text { orthopnea }\end{array}$ & CPB & Operative case & $\begin{array}{l}\text { Mediastinal cystic } \\
\text { teratoma }\end{array}$ & $\begin{array}{c}\text { Severe airway } \\
\text { obstruction }\end{array}$ & $\begin{array}{l}\text { Surgical } \\
\quad \text { resection }\end{array}$ & Discharged \\
\hline Villanueva ${ }^{36}$ & 2014 & $\mathrm{~F} / 63$ & Stridor & CPB & $\begin{array}{l}\text { Until distal } \\
\text { intubation } \\
\text { following } \\
\text { sternotomy }\end{array}$ & $\begin{array}{l}\text { Adenoid cystic } \\
\text { carcinoma }\end{array}$ & $\begin{array}{l}\text { Near complete } \\
\text { airway } \\
\text { obstruction }\end{array}$ & $\begin{array}{l}\text { Surgical } \\
\quad \text { resection }\end{array}$ & Discharged \\
\hline
\end{tabular}


Table continued

\begin{tabular}{|c|c|c|c|c|c|c|c|c|c|}
\hline First author & Year & Age & $\begin{array}{l}\text { Condition } \\
\text { prior to } \\
\text { ECMO }\end{array}$ & $\begin{array}{l}\text { ECMO } \\
\text { mode }\end{array}$ & ECMO duration & Obstruction type & Comments & $\begin{array}{l}\text { Definitive } \\
\text { management }\end{array}$ & Final result \\
\hline Liou $^{41}$ & 2014 & $\mathrm{M} / 76$ & $\begin{array}{l}\text { Respiratory } \\
\text { distress and } \\
\text { stridor }\end{array}$ & VV & Operative case & $\begin{array}{l}\text { Thyroid } \\
\text { carcinoma }\end{array}$ & $\begin{array}{l}\text { 3.8-mm } \\
\text { diameter }\end{array}$ & $\begin{array}{l}\text { Tracheal } \\
\text { resection } \\
\text { and } \\
\text { anastomosis }\end{array}$ & Discharged \\
\hline $\mathrm{Kim}^{45}$ & 2015 & $F / 88$ & $\begin{array}{c}\text { Dyspnea and } \\
\text { orthopnea }\end{array}$ & VV & $\begin{array}{l}2 \mathrm{hr} 20 \mathrm{~min} \\
\text { (operative } \\
\text { case) }\end{array}$ & $\begin{array}{l}\text { Mediastinal } \\
\text { malignant } \\
\text { teratoma }\end{array}$ & $\begin{array}{l}\text { Near complete } \\
\text { obstruction }\end{array}$ & $\begin{array}{l}\text { Resection and } \\
\text { cautery }\end{array}$ & Discharged \\
\hline $\mathrm{Kim}^{50}$ & 2015 & Adult & Dyspnea & VV & Operative case & Tracheal stenosis & & Surgical repair & Discharged \\
\hline $\mathrm{Kim}^{50}$ & 2015 & Adult & Dyspnea & VV & Operative case & Tracheal stenosis & & Surgical repair & Discharged \\
\hline $\mathrm{Kim}^{50}$ & 2015 & Adult & Dyspnea & VV & Operative case & Tracheal stenosis & & Surgical repair & Discharged \\
\hline $\mathrm{Kim}^{50}$ & 2015 & Adult & Dyspnea & VV & Operative case & Tracheal stenosis & & Surgical repair & Discharged \\
\hline $\mathrm{Kim}^{50}$ & 2015 & Adult & Dyspnea & VV & Operative case & Tracheal stenosis & & Surgical repair & Discharged \\
\hline $\mathrm{Kim}^{50}$ & 2015 & Adult & Dyspnea & VV & $\begin{array}{l}\text { Delayed ECMO } \\
\text { weaning due } \\
\text { to } \\
\text { postoperative } \\
\text { ARDS }\end{array}$ & Tracheal stenosis & & Surgical repair & Discharged \\
\hline Dunkman $^{33}$ & 2017 & $\mathrm{M} / 37$ & $\begin{array}{l}\text { Dyspnea and } \\
\text { foreign } \\
\text { body } \\
\text { sensation }\end{array}$ & VV & $\begin{array}{l}46 \min \\
\text { (operative } \\
\text { case) }\end{array}$ & $\begin{array}{l}\text { Endobronchial } \\
\text { schwannoma }\end{array}$ & $\begin{array}{l}\text { Carina } \\
\quad \text { occlusion }\end{array}$ & $\begin{array}{l}\text { Resection of } \\
\text { mass }\end{array}$ & Discharged \\
\hline Fung $^{55}$ & 2017 & $F / 73$ & $\begin{array}{l}\text { Severe } \\
\quad \text { dyspnea }\end{array}$ & VV & Operative case & $\begin{array}{l}\text { Sino-nasal } \\
\text { malignant } \\
\text { melanoma }\end{array}$ & $\begin{array}{l}\text { Near complete } \\
\text { occlusion }\end{array}$ & $\begin{array}{l}\text { Resection of } \\
\text { lesions }\end{array}$ & Discharged \\
\hline Giovacchini $^{34}$ & 2017 & $\mathrm{M} / 37$ & $\begin{array}{l}\text { Biphasic } \\
\text { wheeze }\end{array}$ & VV & Operative case & $\begin{array}{l}\text { Endobronchial } \\
\text { schwannoma }\end{array}$ & $\begin{array}{l}\text { Near complete } \\
\text { mobile } \\
\text { obstruction }\end{array}$ & $\begin{array}{c}\text { Resection of } \\
\text { the lesion }\end{array}$ & Discharged \\
\hline $\mathrm{Natt}^{39}$ & 2017 & $\mathrm{~F} / 53$ & $\begin{array}{l}\text { Respiratory } \\
\text { distress and } \\
\text { stridor }\end{array}$ & VV & Operative case & $\begin{array}{r}\text { Traumatic } \\
\text { tracheal } \\
\text { stenosis }\end{array}$ & $2 \mathrm{~mm}$ diameter & Tracheal stent & Discharged \\
\hline $\operatorname{Tian}^{28}$ & 2017 & $\mathrm{~F} / 60$ & $\begin{array}{l}\text { Severe } \\
\text { dyspnea }\end{array}$ & VV & $97 \mathrm{~min}$ & $\begin{array}{l}\text { Tracheal } \\
\text { adenocarcinoma }\end{array}$ & $\begin{array}{l}\text { Near complete } \\
\text { occlusion }\end{array}$ & $\begin{array}{l}\text { Surgical } \\
\quad \text { resection }\end{array}$ & Discharged \\
\hline
\end{tabular}

$\mathrm{CPB}=$ cardiopulmonary bypass; $\mathrm{ECMO}=$ extracorporeal membrane oxygenation; $\mathrm{LMB}$ = left main bronchus; RMB = right main bronchus; $\mathrm{VA}$ = veno-arterial; $\mathrm{VV}=$ veno-venous

pathology, including tracheal tumours $(31 \%)$, tracheal stenosis $(20 \%)$, and head and neck cancers $(20 \%)$. Cases included in this review had ECMO instigated in a controlled environment prior to airway management, and as such, all reported a favourable patient outcome with all patients surviving to hospital discharge without significant complications.

The first reported successful use of ECMO in the treatment of adult airway obstruction due to thyroid carcinoma was reported by Onozawa et al. ${ }^{29}$ in 1999. Since then, it has been used during a range of surgical procedures involving the respiratory tract to provide gas exchange and hemodynamic support during stenting, tracheotomy, and intubation. ${ }^{58-60}$

Conventional elective management of critical airway obstruction ordinarily involves methods that maintain spontaneous respiration. ${ }^{4,5,7}$ Awake tracheal intubations via either the oral or nasal route, or an awake tracheotomy, are the usual techniques employed. Awake intubation is unsuccessful if the two key reflexes (gag and glottic closure) are not obliterated, usually with local anesthesia, as well as having recognized hazards such as complete airway obstruction occurring during topicalization of the upper airway. ${ }^{61-64}$ Occasionally, during elective surgery for upper airway pathology, jet ventilation is used to maintain oxygenation, ${ }^{65}$ with ventilation being maintained through passive expiration. Nevertheless, in the case of nearcomplete airway obstruction, jet ventilation carries an unacceptably high risk of volutrauma, barotrauma, pneumothorax, hyperventilation, and gastric insufflation. ${ }^{66}$ Recently, active expiration techniques of jet ventilation have also been described that may reduce these risks. ${ }^{65,67}$ 
The CAFG difficult airway guidelines state, "When planning how to approach the anticipated difficult airway, the primary focus should be on ensuring adequate oxygenation and ventilation and not simply on intubating the trachea". 6 Current difficult airway guidelines focus on the management of the anticipated difficult airway using an approach of assessment of the probable success of oxygenation and ventilation; the four fundamental techniques of oxygenation are BMV, SGAs, tracheal intubation, and the front-of-neck airway. Nevertheless, the utility of ECMO in achieving the primary endpoint of oxygenation has not been discussed.

In 2015, Kim et al. assessed the utility of ECMO in the treatment of airway obstruction. ${ }^{21}$ In their institution, 15 patients underwent ECMO for upper airway obstruction due to various pathologies. Based on their analysis they recommended that, when ECMO support is indicated for airway obstruction surgery, with bronchoscopic or chest CT findings determining the tracheal patency to be less than $5 \mathrm{~mm}$, elective insertion of cannulae should be considered.

Typically, the outcomes following the emergency placement of ECMO for rescue of sudden cardiorespiratory arrest are poor, because it takes time to prepare and initiate ECMO. ${ }^{68}$ Cardarelli et al. performed a meta-analysis of studies of adult extracorporeal cardiopulmonary resuscitation initiated for sudden witnessed cardiac arrest. They reported a negative trend in survival when manual CPR lasted $>30 \mathrm{~min}$ without ECMO initiation. ${ }^{69}$ In addition, they reported that the average duration of cardiopulmonary resuscitation before ECMO was approximately $40 \mathrm{~min}$, indicating that performing ECMO swiftly to prevent hypoxic brain injury is not without difficulties. ${ }^{69}$ This point cannot be underestimated. The personnel and equipment, as well as the organizational logistics, required for the safe implementation of ECMO/CPB are critically important. For our patient, we had at least $24 \mathrm{hr}$ to plan for the management. Due to significant cardiovascular comorbidities, and technically challenging cannulae insertion with the patient placed in a semi-upright position, 70 min was taken to initiate ECMO.

The 4th National Audit Project (NAP4) of The Royal College of Anaesthetists and The Difficult Airway Society $^{70}$ reported on major complications of airway events during the management of patients with head and neck pathology. Of the 72 cases reported to the project, ten occurred during induction of anesthesia, and, of these, two patients died as a result of failure to adequately prepare for anticipated difficulties with all four fundamental techniques of oxygenation. These patients were electively anesthetized without consideration of extracorporeal life support for gas exchange. The utility of extracorporeal life support for the management of this patient group was not discussed within the NAP4 document.

Extracorporeal membrane oxygenation is used in many tertiary care centres for acute, severe reversible respiratory or cardiac failure that is refractory to conventional management. Nevertheless, its consideration has not been incorporated into guidelines for the management of ventilatory failure secondary to near complete airway obstruction. As with all oxygenation techniques, ECMO has its limitations. In the context of bridging until the airway is secured, ECMO has very few absolute contraindications. Nevertheless, the use of ECMO is continuously evolving. Clearly, it is essential to involve an ECMO specialist in discussing indications and contraindications in each instance. Long-term ECMO (and long-term intubation) use is associated with complications such as renal failure requiring continuous hemofiltration (52\%), bacterial pneumonia (33\%), bleeding $(33 \%)$, sepsis $(26 \%)$, hemolysis (18\%), liver dysfunction $(16 \%)$, leg ischemia $(10 \%)$, venous thrombosis $(10 \%)$, central nervous system complications (8\%), gastrointestinal bleeding (7\%), aspiration pneumonia $(5 \%)$, and disseminated intravascular coagulation $(5 \%)$. $^{71}$ These complications were all reported following cases in which the duration of ECMO lasted from 5.5 to 9.5 days. The reported incidence of complications with ECMO for short periods is low. ${ }^{23}$

This review focuses on studies utilizing $\mathrm{CPB}$ and ECMO as an elective procedure prior to airway intervention. This review did not include cases in which ECMO was used as a salvage technique or those in which ECMO was used during an emergency; thus, we are limiting the number of cases undergoing ECMO/CPB for all presentations of airway obstruction, which further limits the scope of this review. While all case reports of ECMO/ $\mathrm{CPB}$ in the management of airway obstruction had favourable outcomes, the possibility of positive publication bias and the limited patient scope of this review should be considered.

Extracorporeal oxygenation of systemic blood whilst on ECMO is determined by a combination of factors: the gas exchange capability of the membrane oxygenator, flow rates through the extracorporeal circuit, oxygen uptake within the native lung, and the native cardiac output. Oxygen exchange in the circuit oxygenator occurs across a semipermeable membrane. Diffusion occurs rapidly across the membrane because of a relatively large oxygen concentration gradient. Therefore, within the extracorporeal circuit, the critical factor for oxygen delivery is the contact of the blood within the circuit with the membrane. The greater the volume of blood in contact with the membrane is, the greater the saturation of hemoglobin with oxygen. Therefore, due to the limitation 
of the surface area of the oxygenator, the amount of oxygen provided via the artificial lung is a direct function of the blood flow. ${ }^{72}$

In a VV-ECMO circuit, the membrane oxygenator is in series with the native lung. The improvement in arterial oxygenation in this circuit is due to the increased oxygen saturation of the venous blood flowing through shunt regions of the native lung. The VV-ECMO approach with high flow, even with a very high shunt in the native lung, can provide vital arterial oxygenation. This approach, if the anatomy of the patient (for venous cannulation) had been favourable, would have been the preferred approach for the case presented.

The VA-ECMO approach involves the membrane oxygenator in parallel with the native lung. This circuit involves the drainage of venous blood, oxygenation of the blood, and the subsequent return to the aorta through a cannulated artery. In the setting of complete cardiac failure, there is significantly better systemic oxygenation with this technique compared with the VV-ECMO approach because the artificially oxygenated blood mixes with arterial blood and directly perfuses distal organs. In the presented case, this approach was chosen because of anatomical and technical reasons precluding the use of the VV-ECMO approach. Although this approach provided adequate tissue oxygenation during airway manipulation, it is associated with higher risks of complications related to arterial cannulation and generally higher required levels of systemic anticoagulation. ${ }^{16}$

\section{Conclusions}

While most practitioners are familiar with the four fundamental techniques for oxygenation in airway management, the use of ECMO may not be considered by practitioners working in non-ECMO centres. The $a$ priori use of ECMO is an effective means of providing adequate oxygenation for patients with a severe airway obstruction in which all four fundamental techniques of oxygenation are likely to be unsuccessful. The use of ECMO in tertiary care centres with appropriate resources should be considered in patients with severe (or nearcomplete) airway obstruction secondary to anterior neck or tracheal disease. This approach can provide essential tissue oxygenation while attempts to secure a definitive airway are carried out in a controlled environment.

Conflicts of interest The authors declare no competing interests.

Editorial responsibility This submission was handled by Dr. Hilary P. Grocott, Editor-in-Chief, Canadian Journal of Anesthesia.
Author contributions Gemma Malpas contributed to the study design, literature search, interpretation of data, manuscript preparation, and validation of contents. Orlando Hung contributed to the study design, interpretation of data, manuscript preparation, and validation of contents. Ainslie Gilchrist contributed to the case report and manuscript preparation. Chrison Wong contributed to the case report, literature search, and manuscript preparation. Blaine Kent, Greg Hirsch, and Robert D. Hart contributed to the manuscript preparation.

Funding Support was provided solely from institutional and/or departmental sources.

\section{Appendix Search criteria}

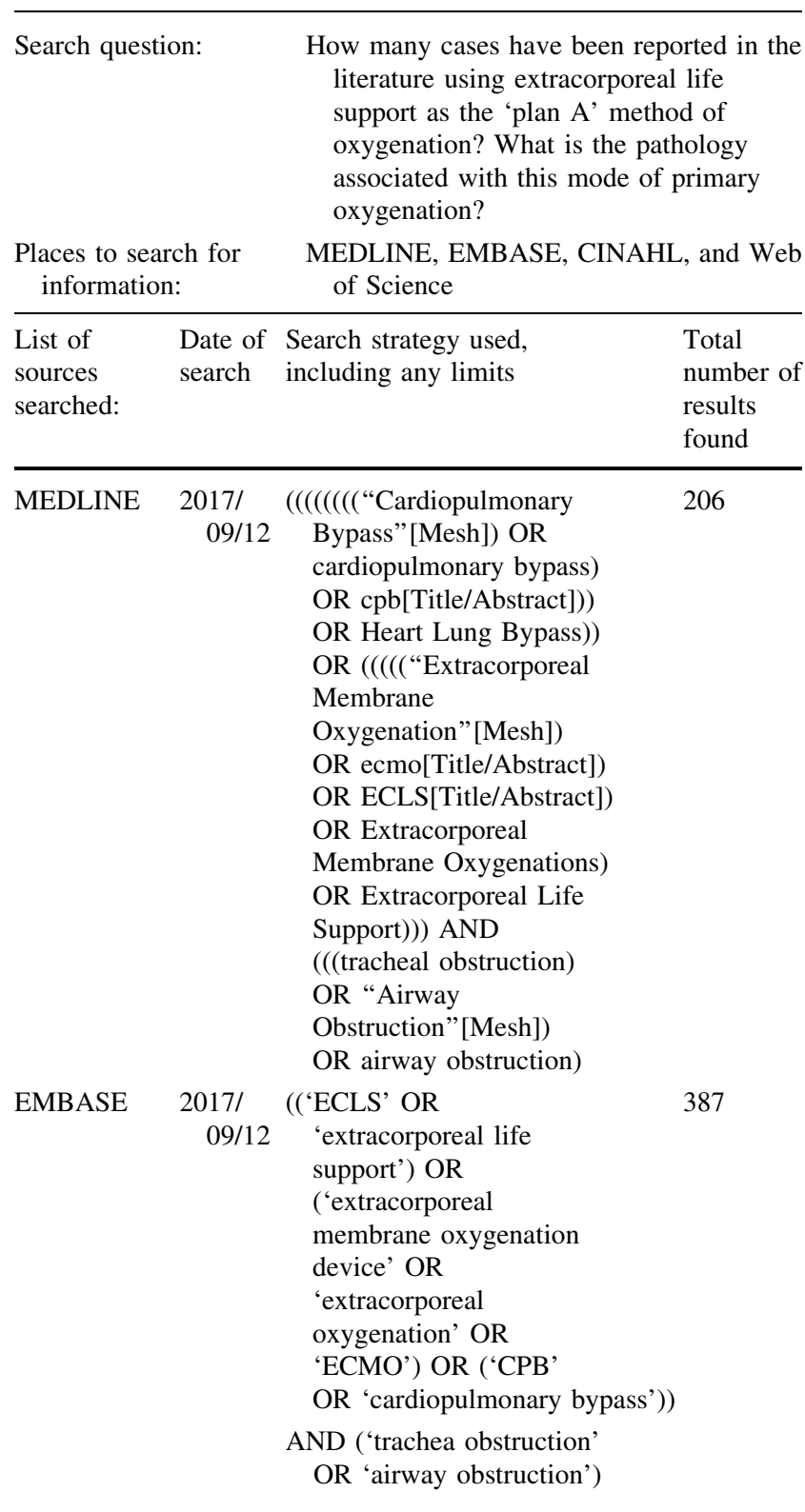


Apendix continued

\begin{tabular}{|c|c|c|c|}
\hline $\begin{array}{l}\text { List of } \\
\text { sources } \\
\text { searched: }\end{array}$ & $\begin{array}{l}\text { Date of } \\
\text { search }\end{array}$ & $\begin{array}{l}\text { Search strategy used, } \\
\text { including any limits }\end{array}$ & $\begin{array}{l}\text { Total } \\
\text { number of } \\
\text { results } \\
\text { found }\end{array}$ \\
\hline CINAHL & $\begin{array}{l}2017 / \\
09 / 12\end{array}$ & $\begin{array}{l}\text { (ECMO OR extracorporeal } \\
\text { membrane oxygenation OR } \\
\text { ECLS OR extracorporeal life } \\
\text { support OR cardiopulmonary } \\
\text { bypass OR CPB) AND } \\
\text { (trachea obstruction OR } \\
\text { airway obstruction) }\end{array}$ & 20 \\
\hline $\begin{array}{l}\text { Web of } \\
\text { Science }\end{array}$ & $\begin{array}{l}\text { 2017/ } \\
09 / 12\end{array}$ & $\begin{array}{l}\text { (ECMO OR extracorporeal } \\
\text { membrane oxygenation OR } \\
\text { ECLS OR extracorporeal life } \\
\text { support OR cardiopulmonary } \\
\text { bypass OR CPB) AND } \\
\text { (trachea obstruction OR } \\
\text { airway obstruction) }\end{array}$ & 171 \\
\hline
\end{tabular}

\section{References}

1. Anonymous. Practice guidelines for management of the difficult airway. A report by the American Society of Anesthesiologists Task Force on Management of the Difficult Airway. Anesthesiology 1993; 78: 597-602.

2. American Society of Anesthesiologists Task Force on Management of the Difficult Airway. Practice guidelines for management of the difficult airway: an updated report by the American Society of Anesthesiologists Task Force on Management of the Difficult Airway. Anesthesiology 2003; 98: 1269-77.

3. Apfelbaum JL, Hagberg CA, Caplan RA, et al. Practice guidelines for management of the difficult airway: an updated report by the American Society of Anesthesiologists Task Force on Management of the Difficult Airway. Anesthesiology 2013; 118: 251-70.

4. Frerk C, Mitchell VS, McNarry AF, et al. Difficult Airway Society 2015 guidelines for management of unanticipated difficult intubation in adults. Br J Anaesth 2015; 115: 827-48.

5. Henderson JJ, Popat MT, Latto IP, Pearce AC; Difficult Airway Society. Difficult Airway Society guidelines for management of the unanticipated difficult intubation. Anaesthesia 2004; 59: 675-94.

6. Law JA, Broemling N, Cooper RM, et al. The difficult airway with recommendations for management-part 1-difficult tracheal intubation encountered in an unconscious/induced patient. Can $\mathbf{J}$ Anesth 2013; 60: 1089-118.

7. Law JA, Broemling N, Cooper RM, et al. The difficult airway with recommendations for management-part 2-the anticipated difficult airway. Can J Anesth 2013; 60: 1119-38.

8. Clark BM, Sprung J, Weingarten TN, Warner ME. Airway management changes in patients with mucopolysaccharidoses: the role of video laryngoscopy. Can J Anesth 2017; 64: 981-2.

9. Duggan LV, Lockhart SL, Romano KR, Weingart SD, Levitan $R M$, Brindley $P G$. Front-of-neck airway meets front-of-neck simulation: improving cricothyroidotomy skills using a novel open-access three-dimensional model and the Airway App. Can J Anesth 2017; 10: 1079-81.

10. Agerstrand $C L$, Bacchetta MD, Brodie D. ECMO for adult respiratory failure: current use and evolving applications. ASAIO J 2014; 60: 255-62.
11. Parekh M, Abrams D, Brodie D. Extracorporeal techniques in acute respiratory distress syndrome. Ann Transl Med 2017; 5: 296.

12. Brodie D, Bacchetta $M$. Extracorporeal membrane oxygenation for ARDS in adults. N Engl J Med 2011; 365: 1905-14.

13. Bartlett RH, Gattinoni L. Current status of extracorporeal life support (ECMO) for cardiopulmonary failure. Minerva Anestesiol 2010; 76: 534-40.

14. Martinez, G, Vuylsteke A. Extracorporeal membrane oxygenation in adults. Continuing Education in Anaesthesia, Critical Care \& Pain 2012; 12: 57-61.

15. Dorson W Jr, Baker E, Cohen ML, et al. A perfusion system for infants. Trans Am Soc Artif Intern Organs 1969; 15: 155-60.

16. Makdisi G, Wang IW. Extra corporeal membrane oxygenation (ECMO) review of a lifesaving technology. J Thorac Dis 2015; 7 : E166-76.

17. Peek GJ, Mugford M, Tiruvoipati R, et al. Efficacy and economic assessment of conventional ventilatory support versus extracorporeal membrane oxygenation for severe adult respiratory failure (CESAR): a multicentre randomised controlled trial. Lancet 2009; 374: 1351-63.

18. Perkins GD, Jacobs IG, Nadkarni VM, et al. Cardiac arrest and cardiopulmonary resuscitation outcome reports: update of the Utstein resuscitation registry templates for out-of-hospital cardiac arrest: a statement for healthcare professionals from a Task Force of the International Liaison Committee on Resuscitation (American Heart Association, European Resuscitation Council, Australian and New Zealand Council on Resuscitation, Heart and Stroke Foundation of Canada, InterAmerican Heart Foundation, Resuscitation Council of Southern Africa, Resuscitation Council of Asia); and the American Heart Association Emergency Cardiovascular Care Committee and the Council on Cardiopulmonary, Critical Care, Perioperative and Resuscitation. Resuscitation 2015; 96: 328-40.

19. Fang ZA, Van Diepen S; Royal Alexandra Hospital and University of Alberta Hospital Cardiac Arrest Teams. Successful inter-hospital transfer for extracorporeal membrane oxygenation after an amniotic fluid embolism induced cardiac arrest. Can J Anesth 2016; 63: 507-8.

20. Rinieri P, Peillon C, Bessou JP, et al. National review of use of extracorporeal membrane oxygenation as respiratory support in thoracic surgery excluding lung transplantation. Eur $\mathbf{J}$ Cardiothorac Surg 2015; 47: 87-94.

21. Kim $C W$, Kim DH, Son BS, et al. The feasibility of extracorporeal membrane oxygenation in the variant airway problems. Ann Thorac Cardiovasc Surg Asia 2015; 21: 517-22.

22. Bricker DL, Parker TM, Dalton ML Jr. Cardiopulmonary bypass in anesthetic management of resection. Its use for severe tracheal stenosis. Arch Surg 1979; 114: 847-9.

23. Hong $Y$, Jo $K W$, Lyu J, et al. Use of venovenous extracorporeal membrane oxygenation in central airway obstruction to facilitate interventions leading to definitive airway security. J Crit Care 2013; 28: 669-74.

24. Liu XY, Liu FY, Wang Z, Chen G. Management and surgical resection for tumors of the trachea and carina: experience with 32 patients. World J Surg 2009; 33: 2593-8.

25. Shiraishi T, Shirakusa T, Hiratsuka M, Yamamoto S, Iwasaki A, Kawahara $K$. Stenting for critical airway stenosis under percutaneous cardiopulmonary support. Jpn J Thorac Cardiovasc Surg 2004; 52: 592-6.

26. Tyagi I, Goyal A, Syal R, Agarwal SK, Tewari P. Emergency cardiopulmonary bypass for impassable airway. J Laryngol Otolol 2006; 120: 687-90.

27. Zhou YF, Zhu SJ, Zhu SM, An XX. Anesthetic management of emergent critical tracheal stenosis. J Zhejiang Univ Sci B 2007; 8: $522-5$. 
28. Tian $F, L i W$, Liang $X$, et al. Application of extracorporeal membrane oxygenation (ECMO) in tracheal tumor resection. Int $\mathrm{J}$ Clin Exp Med 2017; 10: 7244-9.

29. Onozawa H, Tanaka T, Takinami M, Kagaya S, Tanifuji $Y$. Anesthetic management using extracorporeal circulation of a patient with severe tracheal stenosis by thyroid cancer (Japanese). Masui 1999; 48: 658-61.

30. Mehta P, Jokhio S, Wormuth D, Fink G, Lenox R. Extracorporeal membrane oxygenation (ECMO) for management of critical tracheal obstruction. Crit Care Med 2011; 39: 253 (abstract).

31. Wilson RF, Steiger Z, Jacobs J, Sison OS, Holsey C. Temporary partial cardiopulmonary bypass during emergency operative management of near total tracheal occlusion. Anesthesiology 1984; 61: 103-5.

32. Jensen V, Milne B, Salerno T. Femoral-femoral cardiopulmonary bypass prior to induction of anaesthesia in the management of upper airway-obstruction. Can Anaesth Soc J 1983; 30(3 Pt 1): 270-2.

33. Dunkman WJ, Nicoara A, Schroder J, et al. Elective venovenous extracorporeal membrane oxygenation for resection of endotracheal tumor: a case report. A A Case Rep 2017; 9: 97-100.

34. Giovacchini CX, Blough B, Chen L, Wahidi MM. Primary endobronchial schwannoma successfully removed via rigid bronchoscopy on veno-venous extracorporeal membrane oxygenation (VV-ECMO) support. Am J Respir Crit Care Med 2017; 195: A5616.

35. Goyal A, Tyagi I, Tewari P, Agarwal SK, Syal R. Management of difficult airway in intratracheal tumor surgery. BMC Ear Nose Throat Disord 2005; 5: 4.

36. Villanueva $C$, Milder D, Manganas $C$. Peripheral cardiopulmonary bypass under local anaesthesia for tracheal tumour resection. Heart Lung Circ 2015; 24: e86-8.

37. Gao H, Zhu B, Yi J, Ye TH, Huang YG. Urgent tracheal resection and reconstruction assisted by temporary cardiopulmonary bypass: a case report. Chin Med Sci J 2013; 28: 55-7.

38. Chiu $C L$, Teh BT, Wang CY. Temporary cardiopulmonary bypass and isolated lung ventilation for tracheal stenosis and reconstruction. Br J Anaesth 2003; 91: 742-4.

39. Natt B, Knepler $J$ Jr, Kazui T, Mosier JM. The use of extracorporeal membrane oxygenation in the bronchoscopic management of critical upper airway obstruction. J Bronchololgy Interv Pulmonol 2017; 24: e12-4.

40. Jeon HK, So YK, Yang JH, Jeong HS. Extracorporeal oxygenation support for curative surgery in a patient with papillary thyroid carcinoma invading the trachea. J Laryngol Otol 2009; 123: 807-10.

41. Liou JY, Chow LH, Chan KH, Tsou MY. Successful anesthetic management of a patient with thyroid carcinoma invading the trachea with tracheal obstruction, scheduled for total thyroidectomy. J Chin Med Assoc 2014; 77: 496-9.

42. Yang $R, Y u X, M a L, W u F$. Emergency management of a patient with severe airway obstruction resulting from poorly differentiated thyroid carcinoma: a case report. Oncol Lett 2012; 4: 771-4.

43. Belmont MJ, Wax MK, DeSouza FN. The difficult airway: cardiopulmonary bypass-the ultimate solution. Head Neck 1998; 20: 266-9.

44. Rosa P Jr, Johnson EA, Barcia PJ. The impossible airway: a plan. Chest 1996; 109: 1649-50.

45. Weinbroum AA, Kidron A, Mohr R, Kudlik N, Gil Z, Fliss DM. Multidisciplinary management of life-threatening tracheal obstruction. Resuscitation 2005; 64: 115-7.

46. Coles JC, Doctor A, Lefcoe M, Butlin G. A method of anesthesia for imminent tracheal obstruction. Surgery 1976; 80: 379-81.

47. Sendasgupta C, Sengupta G, Ghosh K, Munshi A, Goswami A. Femoro-femoral cardiopulmonary bypass for the resection of an anterior mediastinal mass. Indian J Anaesth 2010; 54: 565-8.
48. Erden IA, Ayhan B, Saylan A, et al. Tracheal stent implantation with extracorporeal circulation (Turkish). Turk Gogus Kalp Damar Cerrahisi Dergisi 2014; 22: 163-7.

49. Said SM, Telesz BJ, Makdisi G, et al. Awake cardiopulmonary bypass to prevent hemodynamic collapse and loss of airway in a severely symptomatic patient with a mediastinal mass. Ann Thorac Surg 2014; 98: e87-90.

50. Kim JJ, Moon SW, Kim YH, Choi SY, Jeong SC. Flexible bronchoscopic excision of a tracheal mass under extracorporeal membrane oxygenation. J Thorac Dis 2015; 7: E54-7.

51. Soon JL, Poopalalingam R, Lim CH, Koong HN, Agasthian T. Peripheral cardiopulmonary bypass-assisted thymoma resection. J Cardiothorac Vasc Anesth 2007; 21: 867-9.

52. Tempe DK, Arya R, Dubey S, et al. Mediastinal mass resection: femorofemoral cardiopulmonary bypass before induction of anesthesia in the management of airway obstruction. J Cardiothorac Vasc Anesth 2001; 15: 233-6.

53. Shao $Y$, Shen $M$, Ding Z, Liang $Y$, Zhang S. Extracorporeal membrane oxygenation-assisted resection of goiter causing severe extrinsic airway compression. Ann Thorac Surg 2009; 88: 659-61.

54. Hicks GL Jr. The impossible intubation-what next? J Cardiovasc Surg (Torino) 1986; 27: 737-9.

55. Fung RKF, Stellios $J$, Bannon RG, Ananda A, Forrest P. Elective use of veno-venous extracorporeal membrane oxygenation and high-flow nasal oxygen for resection of subtotal malignant distal airway obstruction. Anaesth Intensive Care 2017; 45: 88-91.

56. Gourdin M, Dransart C, Delaunois L, Louagie YA, Gruslin A, Dubois $P$. Use of venovenous extracorporeal membrane oxygenation under regional anesthesia for a high-risk rigid bronchoscopy. J Cardiothorac Vasc Anesth 2012; 26: 465-7.

57. Canadian Cancer Society's Advisory Committee. Canadian Cancer Statistics. Government of Canada - Canadian Cancer Society 2016. Available from URL: http://www.cancer.ca/ / media/cancer.ca/CW/cancer\%20information/cancer\%20101/Can adian\%20cancer\%20statistics/Canadian-Cancer-Statistics-2016EN.pdf?la=en (accessed December 2017).

58. Chen A. ECMO-assisted rigid bronchoscopy for tracheal obstruction. J Bronchology Interv Pulmonol 2009; 16: 296-7.

59. Kaneko $T$, Itani $M$, Komasawa $N$, Ueki R, Tatara $T$. Anesthesia for tracheal metal stent management utilizing venovenous extracorporeal life support (Japanese). Masui 2012; 61: 1269-72.

60. Hackner K, Bein T, Kuehnel T, Philipp A, Wittmann S, Graf BM. Elective use of extracorporeal lung assist: prevention of an airway disaster (German). Der Anaesthesist 2010; 59: 1008-12.

61. Shaw IC, Welchew EA, Harrison BJ, Michael S. Complete airway obstruction during awake fibreoptic intubation. Anaesthesia 1997; 52: $582-5$.

62. McGuire G, el-Beheiry H. Complete upper airway obstruction during awake fibreoptic intubation in patients with unstable cervical spine fractures. Can J Anesth 1999; 46: 176-8.

63. Ho AM, Chung DC, To EW, Karmakar MK. Total airway obstruction during local anesthesia in a non-sedated patient with a compromised airway. Can J Anesth 2004; 51: 838-41.

64. White AN, Wong DT, Goldstein CL, Wong J. Cervical spine overflexion in a halo orthosis contributes to complete upper airway obstruction during awake bronchoscopic intubation: a case report. Can J Anesth 2015; 62: 289-93.

65. de Wolf MW, Gottschall R, Preussler NP, Paxian M, Enk D. Emergency ventilation with the Ventrain ${ }^{\circledR}$ through an airway exchange catheter in a porcine model of complete upper airway obstruction. Can J Anesth 2017; 64: 37-44.

66. Welch EH. The hazards of airway surgery. South Afr J Anaesth Analg 2013; 19: 52-4. 
67. Lang SA. Emergency airway management: what are the roles for surgical cricothyroidotomy and the Ventrain ${ }^{\circledR}$ device? Can J Anesth 2016; 63: 997-8.

68. Azher I, Buggy D, Carton E. Should ECPR be attempted following refractory cardiac arrest secondary to airway obstruction by a mediastinal mass? Acta Anaesthesiol Belg 2017; 68: 39-41.

69. Cardarelli MG, Young AJ, Griffith B. Use of extracorporeal membrane oxygenation for adults in cardiac arrest (E-CPR): a meta-analysis of observational studies. ASAIO J 2009; 55: 581-6.

70. Patel A, Pearce A, Pracy P. Head and neck pathology. In: Cook T, Woodall N, Frerk C (Eds). $4^{\text {th }}$ National Audit Project of The
Royal College of Anaesthetists and The Difficult Airway Society. Major complications of airway management in the United Kingdom. Report and Findings March 2011: 143-54. Available from URL: https://www.rcoa.ac.uk/system/files/CSQ-NAP4-Full. pdf (accessed December 2017).

71. Zangrillo A, Landoni $G$, Biondi-Zoccai $G$, et al. A meta-analysis of complications and mortality of extracorporeal membrane oxygenation. Crit Care Resusc 2013; 15: 172-8.

72. Chauhan S, Subin S. Extracorporeal membrane oxygenation, an anesthesiologist's perspective: physiology and principles. Part 1. Ann Card Anaesth 2011; 14: 218-29. 